\section{Revista Brasileira de Administração Científica}

Brazilian Journal of Scientific Administration

Abr a Jun 2020 - v.11 - n.2

\title{
Reflexões sobre aprendizagem e inovação em organizações: uma visão acerca dos aspectos temporais e contextuais
}

Aprendizagem e inovação em organizações são processos complexos e apresentam uma vasta literatura fundamentada em diferentes conceitos e perspectivas. 0 tempo e o contexto organizacional aparecem como elementos capazes de exercer influência na aprendizagem, o que por sua vez exerce influência na inovação, já que está é resultante do processo de aprendizagem. Logo, é a partir do processo de aprendizagem que novos conhecimentos surgem, impulsionando mudanças tecnológicas e inovações, proporcionando diferenciação e competitividade para as organizações. Nessa linha, torna-se relevante articular por meio de um ensaio teórico, os conceitos que compõem a aprendizagem e a inovação, bem como os aspectos e as características contextuais e temporais envolvidos nesses processos, sendo este o objetivo principal deste ensaio. Para alcançar tal objetivo, cada construto é definido, considerando suas especificidades, bem como são apresentadas as diferentes inserções teóricas já propostas sobre a temática. Por fim, são indicadas sugestões para pesquisas futuras que podem vir a elucidar questões que aparecem como temas obscuros ou pouco tratados, sobretudo relativos ao tempo e ao contexto e suas relações com a aprendizagem e a inovação. A relevância desta proposta consiste em demonstrar a necessidade de avançar na compreensão de elementos que compõem as bases teóricas da temática proposta.

Palavras-chave: Aprendizagem em organizações; Processo de Inovação; Tempo; Contexto.

\section{Reflections on learning and innovation in organizations: a view about temporal and contextual aspects}

\begin{abstract}
Learning and innovation in organizations are complex processes and present a vast literature based on different concepts and perspectives. Time and the organizational context appear as elements capable of influencing learning, which in turn influences innovation, since it results from the learning process. Therefore, it is from the learning process that new knowledge emerges, driving technological changes and innovations, providing differentiation and competitiveness for organizations. In this line, it becomes relevant to articulate through a theoretical essay, the concepts that make up learning and innovation, as well as the contextual and temporal aspects and characteristics involved in these processes, this being the main objective of this essay. To achieve this goal, each construct is defined considering its specificities, as well as the different theoretical inserts already proposed on the theme. Finally, suggestions for future research are suggested, which may elucidate issues that appear as obscure or poorly treated themes, especially relating to time and context and their relationship with learning and innovation. The relevance of this proposal is to demonstrate the need to advance the understanding of elements that make up the theoretical bases of the proposed theme.
\end{abstract}

Keywords: Learning in organizations; Innovation Process; Time; Context.

Topic: Inovação Tecnológica

Reviewed anonymously in the process of blind peer.
Received: 20/03/2020

Approved: 02/04/2020
Simone Costa Silva (iD

Universidade Federal de Pernambuco, Brasil http://lattes.cnpq.br/5339837796115908 http://orcid.org/0000-0003-1624-2912

monyadm@yahoo.com.br

Marcos André Mendes Primo (iD

Universidade Federal de Pernambuco, Brasil http://lattes.cnpq.br/0801402521183018 http://orcid.org/0000-0001-8126-9252

marcosprimo0@gmail.com

Eduardo de Aquino Lucena (iD)

Universidade Federal de Pernambuco, Brasil http://lattes.cnpq.br/4896424955173386 http://orcid.org/0000-0001-8441-7337 eaqlucena@yahoo.com.br

\author{
Jéssica Micaelly Santana do Nascimento Silva (iD) \\ Universidade Federal da Paraíba, Brasil \\ http://lattes.cnpq.br/6942231983740310 \\ http://orcid.org/0000-0002-0453-9324 \\ jessica nascimento21@hotmail.com

\section{Eunice Ferreira Carvalho (iD} \\ Universidade Federal da Paraíba, Brasil \\ http://lattes.cnpq.br/0787250920518181 \\ http://orcid.org/0000-0002-2333-1342 \\ elocarvalho21@gmail.com
}

Referencing this:

SILVA, S. C.; MENDES PRIMO, M. A.; LUCENA, E. A.; SILVA, J. M. S. N.; CARVALHO, E. F.. Reflexões sobre aprendizagem e inovação em organizações: uma visão acerca dos aspectos temporais e contextuais. Revista Brasileira de Administração Científica, v.11, n.2, p.139-155, 2020. DOI: http://doi.org/10.6008/CBPC2179-684X.2020.002.0010 


\section{INTRODUÇÃO}

A inovação tem, ao longo do tempo, chamado a atenção de muitos pesquisadores e estudiosos que têm abordado essa temática a partir de distintas perspectivas, tais como Economia, Psicologia, Engenharia, Estudos Organizacionais, entre outros (FAGERBERG, 2004). Tal interesse pelo tema pode ser resultado da relação, já evidenciada em estudos anteriores, entre inovação e desempenho empresarial (JIMÉNEZ-JIMÉNEZ et al., 2011), bem como pela relevância que a área apresenta por estar associada à conquista de diferenciação e vantagem competitiva (ROSSETTO et al., 2016). Isso coloca a capacidade para inovar não como uma escolha, mas como um imperativo para aquelas organizações que desejam permanecer competitivas no mercado (DOUGHERTY, 2004; BESSANT et al., 2009).

Inicialmente, os estudos sobre inovação tinham como foco a descrição de quais inovações surgiam e por quem eram produzidas. Essa visão tradicional tendia a enfatizar a criação e a distribuição de artefatos físicos (SWAN et al., 2003) e com o passar do tempo resultou em uma série de tipologias e classificações. Passou-se a falar em inovação técnica e administrativa (VAN DE VEN, 1986); inovação de produto, de processo, posição e paradigma (TIDD et al., 2008); inovação tecnológica e não tecnológica, radicais e incrementais (FREEMAN et al., 1998).

Essa ênfase na inovação como um artefato físico foi sendo transformada e transferida para o processo de inovação. Vista como processo, a inovação envolve a geração e a adoção de novas ideias por pessoas que se engajam em transações com outros indivíduos em um determinado contexto, o que implica que a inovação não é uma atividade individual, é um compartilhamento coletivo (VAN DE VEN, 1986). Além disso, a inovação como um processo envolve etapas que se seguem, bem como recursos, atividades e pessoas. Portanto, o processo de inovação parte do indivíduo, mas envolve os grupos e a sociedade, formando uma rede que tem como base o conhecimento (TORNATZKY et al., 1990). Com isso, a ênfase foi transferida da inovação como um artefato para uma visão baseada no conhecimento (SWAN et al., 2003).

Nessa perspectiva, é possível afirmar que o processo de inovação envolve, ao menos, três etapas ou subprocessos: invenção, desenvolvimento e implementação (GARUD et al., 2013). Durante a invenção há a criação do conhecimento; no desenvolvimento, também denominado difusão, ocorre a objetificação desse conhecimento e a implementação envolve a apropriação do conhecimento (SWAN et al., 2003; GARUD et al., 2013). Cabe destacar a necessidade de não ver o processo de inovação como algo engessado, tendendo a visualizar tal fenômeno como um processo linear, quando no dia-a-dia das organizações as coisas podem não acontecer desta forma, etapas do processo de inovação podem retroagir, ou ainda ocorrerem concomitantemente, entre outras possibilidades.

Além disso, a introdução de uma inovação não acontece no vácuo, ela envolve toda uma estrutura operacional e gerencial, assim como rotinas, procedimentos e um sistema de relacionamentos que perpassa os diferentes níveis em que as inovações se desdobram, ou seja, o nível micro que envolve o contexto dentro da empresa, definido como intraorganizacional, o nível meso, entre a empresa e outras organizações, neste caso, interorganizacional e, nível macro, envolvendo toda uma comunidade, setor, região e até mesmo um 
país (FAGERBERG, 2004; TIGRE, 2006; GARUD et al., 2013).

Nesse sentido, o processo de inovação dirige a necessidade de interação da empresa com múltiplos atores intra e interorganizacionais, incorrendo em diversas rotinas e atividades dedicadas a mobilizar, coordenar e integrar os recursos e atores internos com os atores externos à empresa, para explorar oportunidades tecnológicas e de mercado, alinhadas às prioridades estratégicas corporativas (TIDD et al., 2008). Assim sendo, a inovação é de natureza tanto social quanto organizacional, ou seja, está atrelada à relação entre os indivíduos, mas também com outros fenômenos organizacionais, tais como a cultura, gestão de conflitos, processo de comunicação, entre outros (BAILEY et al., 2003).

A inovação é, portanto, um processo complexo, isto é, envolve a combinação ou interação entre elementos heterogêneos e que têm natureza não linear (GARUD et al., 2011b). Trata-se de um processo em curso, indeterminado e aberto, com os atores mobilizando eventos do passado, presente e futuro para perseguir suas iniciativas e projetos (GARUD et al., 2016). Assim, a inovação envolve aprender a encontrar a solução mais apropriada para o problema de gerenciar consistentemente esse processo, e fazê-lo da maneira mais adequada às circunstâncias particulares nas quais a organização se encontra (BESSANT, 2003).

Dessa forma, o aprendizado configura-se como um processo pelo qual a replicação e à experimentação permitem que as atividades sejam executadas de forma mais eficiente e que novas oportunidades de produção sejam identificadas (TEECE, 2007). Logo, é a partir do processo de aprendizagem que novos conhecimentos surgem, impulsionando mudanças tecnológicas e inovações.

Tsoukas (2009) expõe que inovação e aprendizagem ocorrem a partir do surgimento de novo conhecimento organizacional. Quando esse conhecimento é desenvolvido em novos produtos ou processos, tem-se a inovação, já a aprendizagem se dá ao incorporar esse mesmo conhecimento em novas ações. Nesse sentido, a inovação pode ser vista como sendo resultado do processo de aprendizagem, e ainda, pode-se afirmar que a inovação permite materializar ou tornar palpável o conhecimento gerado por meio do processo de aprendizagem.

É importante ressaltar que ao estudar aprendizagem, é possível ter foco sobre diferentes níveis de análise em que essa ocorre, quais sejam: no nível individual, grupal ou organizacional. No caso da aprendizagem no nível organizacional, "embora os pesquisadores a tenham definido de diferentes maneiras, o núcleo da maioria das definições é que a Aprendizagem Organizacional (AO) é uma mudança na organização que ocorre quando a mesma adquire experiência" (ARGOTE et al., 2011). Assim, a aprendizagem denominada de organizacional é aquela que se verifica em integrantes da organização, os quais, no cerne de seus cargos e funções, engajam-se na prática do trabalho organizacional (ELKJAER, 2003). Tal abordagem é sintetizada como a metáfora da aquisição.

Por outro lado, tem sido apontado que o aprendizado ocorre por meio da participação dos indivíduos em comunidades de prática, iniciando-se nas atividades organizacionais concretas. Tal abordagem é conhecida como metáfora da participação, nessa, a AO torna-se uma atividade social, pois o aprendizado passa a ser inserido no dia-a-dia das organizações como algo que faz parte da atividade humana, não podendo ser evitada (ELKJAER, 2004). 
Como uma alternativa para a superação da dicotomia indivíduo-ambiente existente no campo da aprendizagem, Elkjaer (2004; 2013) propõe uma "terceira via" - a metáfora da investigação. O cerne desta metáfora é a adoção do pensamento como um instrumento na aprendizagem e a percepção da aprendizagem como um processo social (ELKJAER, 2003; 2004; 2013). Inclui-se uma compreensão de que há uma influência e formação mútua entre organização e indivíduos (ELKJAER, 2004). A partir dessa perspectiva, aprendizes, de uma forma ou de outra, podem ser vistos como construindo sua compreensão a partir da ampla variedade de materiais que incluem ambiente social, circunstâncias físicas e as histórias e relações sociais das pessoas envolvidas (BROWN et al., 1991).

Logo, a aprendizagem ocorre em um contexto que abrange a organização e o ambiente onde ela está inserida, incluindo elementos de fora das fronteiras da organização, como competidores, clientes, fornecedores, outras instituições e reguladores, bem como elementos internos da organização, como estrutura, cultura, tecnologia, identidade, memória, objetivos, incentivos e estratégia (ARGOTE et al., 2011). Estudar aprendizagem envolve, pois, explorar os contextos específicos das atividades e práticas sociais em que a mesma pode ocorrer (BRANDI et al., 2011). Nesse sentido, conhecer aspectos do contexto que permeiam a aprendizagem e como esse afeta a aprendizagem organizacional (ARGOTE et al., 2011) é fundamental para melhor compreendê-la.

Além disso, "aprendizagem organizacional é um processo que ocorre sobre o tempo" (ARGOTE et al., 2011). A natureza da estrutura temporal dominante influencia o progresso da aprendizagem e a ocorrência de descontinuidades, determinando momentos de mudança organizacional (BERENDS et al., 2010). Garud et al. (2011a) defendem a tese de que a aprendizagem a partir de experiências incomuns pode ser um processo gerador, com a aprendizagem ocorrendo de tal maneira que, em qualquer momento, experiências incomuns passadas podem ser mobilizadas para moldar, mas não determinar respostas a experiências incomuns atuais e futuras. Esses momentos de experiências incomuns, raros ou não usuais representam desafios únicos de aprendizagem (BECK et al., 2009) e estão baseados no que Berends et al. (2010) denominam estrutura temporal, que pode ser fundamentada em diferentes concepções de tempo, o que inclui o tempo cronológico e o tempo evento, sendo o primeiro determinado pelo relógio e o calendário, e o segundo pela ocorrência de eventos significantes.

Da dinâmica temporal surge, assim, "um aspecto central da aprendizagem" (LERVIK et al., 2010). No entanto, mesmo com a impossibilidade de 'escapar' do tempo em relação à vida dos indivíduos e à vida das organizações em particular, grande parte dos acadêmicos de gestão tende a excluir essa temática, ou reduzila a um efeito de pouca importância, compactando-a em variáveis que não são capazes de demonstrar o fluxo real das coisas (LANGLEY et al., 2013; LANGLEY et al., 2010).

Portanto, destaca-se que tempo e contexto se configuram como aspectos chaves quando se deseja estudar temas com perspectiva de processo, a exemplo da aprendizagem e da inovação. É necessário avançar na compreensão dos elementos que os compõem, debruçando-se sobre as questões relativas ao tempo e seus eventos marcantes, além do contexto onde ocorrem esses fenômenos. Para tal, o presente trabalho tem como objetivo principal articular por meio de um ensaio teórico os conceitos que compõem a 
aprendizagem e a inovação, bem como os aspectos e as características contextuais e temporais envolvidos nesses processos.

\section{METODOLOGIA}

\section{Inovação e processo de inovação}

A inovação definida como as novas combinações dos recursos já existentes não é um fenômeno novo, visto que, mesmo antes de Schumpeter - considerado o 'Pai da Inovação' - outros estudiosos já falavam no tema, mesmo sem citar tal termo. No entanto, contribuição substancial sobre o tema inovação é atribuído a Joseph Schumpeter, quem fala de inovação costuma citá-lo (SZMRECSÁNYI, 2002). Suas contribuições foram principalmente em destacar a importância do progresso técnico como motor da dinâmica capitalista, apresentando conceitos chaves como invenção, inovação e difusão. Szmrecsányi (2002) acrescenta ainda que ele foi responsável pela categorização da inovação, ao afirmar que, além de novos mercados, as inovações compreenderiam novos bens, novos métodos de produção, novas fontes de ofertas e novas formas organizacionais. Mas, muito embora esse precursor conceda destaque à inovação em seu modelo de desenvolvimento econômico, ele acaba não examinando como ocorre o processo de geração e difusão tecnológica em si. O que só ocorreu a partir de estudos que o sucederam.

Posteriormente, a abordagem de Schumpeter recebeu a atenção de outros economistas denominados de neo-schumpeterianos - entre os quais é possível citar Freeman (1974), Nelson et al. (1977 e 1982), Rosenberg (1982), Dosi (1984) - estes propõem que "a inovação não é aleatória, ela surge de uma série de atos decorrentes do processo de busca, experimentação e imitação. Ela está no centro do crescimento da firma e da geração de lucro puro" (SOUZA, 2005).

Ao tentar conceituar a inovação, cabe expor que não existe um consenso na literatura sobre isso. De forma mais sucinta, Van de Ven et al. (1995) afirmam que "inovação é a introdução de uma ideia nova". Enquanto Fagerberg (2004) faz uma distinção entre invenção e inovação. Em que a invenção é a primeira ocorrência de uma ideia para um novo produto ou processo, já a inovação é a primeira tentativa para executar isso na prática.

Sob o olhar organizacional, a inovação pode ser conceituada como a capacidade da organização fazer algo novo, que agregue valor e proporcione vantagem competitiva. Essa capacidade é impulsionada pela habilidade de estabelecer relações, detectar oportunidades e tirar proveito destas (TIDD et al., 2008). Nesta perspectiva, toda e qualquer novidade inserida no contexto a que se refere, pode ser considerada uma inovação.

Pesquisadores frequentemente usam os termos exploration e exploitation para direcionar a distinção entre mudanças radicais e incrementais, respectivamente. Assim, exploration implica no comportamento de busca, descoberta e experimentação, estando relacionada à inovação radical. Já exploitation caracteriza-se pelo refinamento, implementação, eficiência, produção e seleção, e se refere à inovação incremental (PENG et al., 2008). 
As inovações incrementais abrangem melhorias feitas no design ou na qualidade dos produtos, aperfeiçoamentos em layout e processos, novos arranjos logísticos e organizacionais e novas práticas de suprimentos e vendas (FREEMAN et al., 1998). Elas não derivam necessariamente de atividades de Pesquisa e Desenvolvimento (P\&D), sendo mais comumente resultantes do processo de aprendizado interno e da capacitação acumulada (TIGRE, 2006). Por outro lado, as inovações radicais, correspondem à introdução de produtos, serviços ou processos totalmente novos no mercado e estão fortemente relacionadas com as atividades de P\&D (FREEMAN et al., 1998).

A inovação também pode ser categorizada como inovação de produto, de processo, posição e paradigma (TIDD et al., 2008). Ou ainda, como técnica (novas tecnologias, produtos e serviços) e administrativa (novos processos, políticas e formas organizacionais) (VAN DE VEN, 1986). Além desses tipos de inovações, há também as mudanças de sistemas tecnológicos, que dizem respeito a mudanças de longo alcance na economia as quais geralmente incluem inovações radicais e incrementais de produtos e processos, e mudanças de paradigma técnico-econômico que diz respeito às mudanças que afetam o comportamento de praticamente toda a economia (FREEMAN et al., 1998).

É perceptível que, muito mais do que apresentar diversas classificações ou categorias para as inovações, a literatura as divide pelo grau de mudança proporcionado - radical ou incremental - ou pelo resultado gerado - produto, serviço, processo, organizacional. No entanto, pensa-se que mais importante do que classificações e categorias, o real valor da inovação consiste naquilo que ela pode proporcionar aos indivíduos e as organizações.

Dessa forma, melhorar produtos e processos existentes e desenvolver novos produtos e processos podem ambos ser considerados como inovação e se configuram como condição fundamental para buscar a sobrevivência organizacional. Nesta perspectiva, a inovação inclui implementação de novas ideias ou mudanças, sejam grandes ou pequenas, e têm o potencial de contribuir para o alcance dos objetivos organizacionais (PENG et al., 2008). Pois, conforme expõe Fagerberg (2004), o que se pensa como uma única inovação é frequentemente resultado de um lento processo envolvendo muitas inovações interrelacionadas. Logo, mais importante que buscar conceitos e classificações para o termo inovação, o que realmente importa ao se aproximar deste assunto é conhecer e explanar como acontece. Nesse sentido, é possível afirmar que ao longo do tempo os estudos sobre inovação partiram da descrição de quais inovações surgiam e quem as produzia, para um olhar sobre a inovação como um processo.

Sobre processo, Van De Ven (1992) propõe três significados para o termo: a) “Uma lógica que explica uma relação causal entre variáveis dependentes e independentes; b) Uma categoria de conceitos ou variáveis que se refere às ações de indivíduos ou organizações; c) Uma sequência de eventos que descreve como as coisas mudam ao longo do tempo". Diante do proposto pelo autor e tendo em vista a intenção deste trabalho, o significado aceito para o termo processo será o terceiro conceito, ou seja, o processo como uma perspectiva histórica com foco numa sequência de incidentes, atividades e estágios que giram em torno de um assunto principal existente (VAN DE VEN, 1992).

Portanto, processo de inovação consiste em motivar e coordenar pessoas para desenvolver e 
implementar novas ideias por engajamento em transações (ou relacionamentos) com outros e fazer as adaptações necessárias para alcançar resultados desejados dentro de um contexto de mudança organizacional (VAN DE VEN et al., 1995). Ou ainda, pode ser definido como "as atividades combinadas que levam a produtos e serviços novos e comercializáveis, ou a novos sistemas de produção e distribuição" (BURGELMAN et al., 2012). A inovação tecnológica gerida como processo pode ser caracterizada como um processo multifacetado que envolve a integração de várias funções da empresa e de atores externos (OECD, 2005).

Uma crítica que se faz a essa visão processual da inovação é a tendência que se tem de visualizar tal fenômeno como um processo linear, quando no dia-a-dia das organizações as coisas podem não acontecer desta forma. Na vida diária, etapas do processo de inovação podem não ocorrer, podem retroagir, ou ainda ocorrerem concomitantemente, entre outras possibilidades.

Seguindo-se, destaca-se que o processo de inovação dirige a necessidade de interação, comunicação da empresa com múltiplos atores intra e interorganizacionais, além de incorrer em diversas rotinas e atividades dedicadas a esse fim. Como já destacado na literatura, inclusive por Schumpeter, as tecnologias são físicas e sociais, portanto, não se limitam a uma questão de engenharia produtiva. Especificamente no caso de Schumpeter, entende-se que, apesar da ênfase dedicada às questões tecnológicas, o autor caracterizou-se também pelo entendimento de que o contexto social afeta e é afetado pelas inovações (PEREIRA et al., 2015).

Sobre isso, Freeman (1982) reitera que o mais importante em termos de crescimento econômico, investimento e emprego não é quando surge a inovação, mas a difusão desta, quando a mesma se espalha e os imitadores começam a perceber o potencial do novo produto ou processo e começam a investir com mais ênfase na tecnologia. Já Rogers (1983) ao definir a difusão como "o processo pelo qual uma inovação é comunicada através de certos canais ao longo do tempo entre os membros de um sistema social", acaba por ratificar tal perspectiva.

A necessidade de abrir-se e preocupar-se com o ambiente onde as inovações são inseridas conduz à concepção interativa do processo de inovação, dentro desse entendimento, à empresa cabe não somente analisar o ambiente competitivo que a cerca, mas também buscar conhecimentos sobre como gerenciar seus recursos e capacidades para prover inovações; isso pode ser alcançado a partir do gerenciamento de forma sistemática, com processos claros e ferramentas adequadas (FUCK et al., 2012).

O gerenciamento da inovação tecnológica busca mobilizar, coordenar e integrar os recursos e atores internos com os atores externos à empresa, para explorar oportunidades tecnológicas e de mercado, alinhadas às prioridades estratégicas corporativas (TIDD et al., 2008). Mas, apesar de sua natureza social e relevância que apresenta para as organizações, segundo Van de Ven et al. (1995), poucas pesquisas sistemáticas têm sido conduzidas para examinar como as inovações se desenvolvem ao longo do tempo, bem como poucas teorias de processo adequadamente explanam a sequência de eventos no processo de inovação. Além disso, o tema ainda é pouco explorado em conjunto com outras temáticas, a exemplo da aprendizagem, que pouco se busca vincular e relacionar conhecimentos, sobretudo por meio de pesquisas 
qualitativas (ARAÚJO et al., 2015).

Neste sentido, uma vez que há importância do tema para a competitividade organizacional, na construção de um ambiente mais favorável às atividades inovadoras, parece promissor a necessidade de conhecer os pilares que sustentam a base teórica da área, bem como investigar empiricamente como esse fenômeno ocorre nas organizações. Isso pode permitir compreender o passado e engendrar esforços conjuntos que permitam atender às necessidades do presente, buscando soluções para as questões atuais e futuras.

\section{DISCUSSÃO TEÓRICA}

\section{Aprendizagem organizacional}

A literatura sobre Aprendizagem Organizacional ( $\mathrm{AO}$ ) nos estudos em administração tem apresentado, em seus mais de 50 anos de legado, um aumento não apenas em quantidade, mas também em qualidade, ao mesmo tempo em que se ramificou e passou a incluir insights de outras ciências, apontando novas dimensões para a temática (ANTONELLO et al., 2010). Com isso, a AO passou a ser permeada por muitos conceitos e teorias e apresentar distintas concepções.

$\mathrm{Na}$ década de 1960, os estudos sobre aprendizagem nas organizações surgiram a partir das investigações de Cyert e March, mas foi a partir da década de 90 que pesquisas nesta área se desenvolveram rapidamente, passando a abordar a eficiência ou o processo social da aprendizagem independentemente, $\mathrm{o}$ que aumentou a dificuldade dessas duas abordagens se comunicarem (EASTERBY-SMITH et al., 2011).

Aprendizagem organizacional, do ponto de vista acadêmico, pode ser conceituada como o estudo do processo de aprendizagem dentro das organizações, buscando conhecer as nuances deste fenômeno (EASTERBY-SMITH et al., 2011). É um tópico inerentemente interdisciplinar nos estudos organizacionais, ao mesmo tempo em que, tanto recebe influência quanto contribui para o desenvolvimento de uma variedade de campos, incluindo teoria e comportamento organizacional, psicologia social e cognitiva, sistemas de informação, entre outros (ARGOTE et al., 2011). Do ponto de vista prático, acrescentam os autores, a habilidade para aprender e se adaptar são críticas para o desempenho e o sucesso de longo prazo das organizações.

Buscando uma conceituação, pode-se dizer que aprendizagem organizacional significa o processo de melhorar as ações através de um melhor entendimento ou conhecimento (FIOL et al., 1985). E ainda, é um processo social de indivíduos participando de práticas coletivas situadas (POPOWA-NOWAK et al., 2015), na qual os indivíduos e as organizações são vistos como mutuamente constituídos (ELKJAER, 2009). Em acréscimo, Knipfer et al. (2013) consideram esse fenômeno como sendo resultado de complexas interações entre a aprendizagem individual e coletiva que são ligadas por processos sociocognitivos.

Além disso, AO é fortemente inter-relacionada com o desenvolvimento de novas práticas, com alguma mudança no conhecimento de seus empregados, sobretudo mudanças consideradas relevantes e que contribuem para o alcance dos objetivos organizacionais existentes ou permitem a geração de novos 
objetivos (KNIPFER et al., 2013).

Nesse sentido, há divergência entre o pensamento desses autores supracitados com o que propõem Fiol et al. (1985) ao afirmarem que "as mudanças no comportamento podem ocorrer sem associação de nenhum desenvolvimento cognitivo. Similarmente, o conhecimento pode ter ocorrido sem nenhuma mudança no comportamento". É o que também propõem Hernes et al. (2012) ao defenderem que "a aprendizagem organizacional acontece não apenas pela adaptação aos problemas, mas também pelo entendimento do que ocorre durante condições estáveis". Com isso, o conhecimento adquirido não é tanto para responder aos desafios e surpresas do ambiente, mas para aumentar o conhecimento sobre o contexto por meio da aprendizagem organizacional (HERNES et al., 2012).

Assim, para fins de condução do presente estudo, a Aprendizagem Organizacional é entendida como um processo social de gerar novos conhecimentos e fornecer mais racionalidade às práticas organizacionais, por meio da interação entre indivíduos e o contexto onde estão inseridos. É por meio da aprendizagem que a organização pode escolher cursos de ação de forma mais consciente, não apenas como resposta às pressões do ambiente, mas por compreender quais escolhas podem ser feitas, quais decisões podem ser tomadas, inclusive a decisão de não mudar.

Brandi et al. (2011) afirmam que num primeiro momento grande parte da literatura em AO foi inspirada na teoria de aprendizagem individual, a partir da qual a aprendizagem é idêntica ao aprimoramento de modelos mentais individuais e acontece quando indivíduos adquirem informação e conhecimento que guia seu comportamento e, por consequência, o comportamento da organização - metáfora da aquisição. Nessa perspectiva, o conhecimento é criado pelos indivíduos, com a organização dando suporte ou provendo o contexto para que se crie tal conhecimento (NONAKA, 1994). Posteriormente, o conhecimento que o indivíduo adquire deve ser incorporado em uma variedade de repositórios como ferramentas, rotinas, valores, redes sociais e sistemas de memórias para tornar-se organizacional (ARGOTE, 2011).

Por outro lado, o fenômeno da aprendizagem organizacional passou a ser abordado a partir da perspectiva da teoria da aprendizagem social. Nessa, a aprendizagem é resultado da participação em processos sociais, com os atores transformando suas relações com outros indivíduos enquanto compartilham atividades (BRANDI et al., 2011). Neste caso, conhecimento é resultado de um processo de produção ativa determinado pela participação em comunidade de prática - metáfora da participação (ELKJAER, 2009).

Há ainda a perspectiva da aprendizagem organizacional fundamentada nos estudos de Dewey e do pragmatismo em que o conceito de aprendizagem está baseado na noção de experiência, não havendo somente a ação ou a prática, mas também o agir e o pensar - 'terceira via' ou metáfora da investigação. Assim, a ação reflexiva é sempre criada a partir de situações particulares ou problemas, sendo possível transferir experiências de uma situação para outra com condições idênticas. Além disso, no pragmatismo é impossível separar o indivíduo do social, do contexto e/ou da organização (ELKJAER, 2009; BRANDI et al., 2011).

Com base no que propõem Popova-Nowak et al. (2015), a ênfase no indivíduo, na eficiência ou no processo social da aprendizagem fez surgir quatro paradigmas para a $A O$, quais sejam: funcionalista, 
construcionista, pós-moderno e crítico. Em cada uma dessas diferentes perspectivas teóricas para compreensão da aprendizagem organizacional, são enquadrados diferentes autores que contribuíram para o seu desenvolvimento com contribuições chaves de acordo com suas tradições teóricas (PAWLOWSKY, 2001).

Nesse sentido, o paradigma funcionalista, dominante em estudos organizacionais e em aprendizagem organizacional, tem como principais abordagens de AO a comportamental, a cognitiva e a baseada na ação social. Além disso, considera os indivíduos como agentes-chaves em coletar, interpretar, disseminar, estocar e reter informação dentro das organizações. Nesse paradigma AO é sinônimo de processamento de informação (POPOVA-NOWAK et al., 2015).

Já no paradigma construcionista, o conhecimento é socialmente construído e baseado na prática. Aprendizagem, nesse sentido, não ocorre somente pela aquisição do conhecimento, mas pela participação dos indivíduos em práticas coletivas. Neste caso, a AO ocorre pela prática organizacional como resultado da interação entre indivíduos e estruturas organizacionais (POPOVA-NOWAK et al., 2015).

Popova-Nowak et al. (2015) expõem, ainda, que a visão pós-moderna enfatiza a AO baseada no conhecimento, poder, linguagem e discurso. Tem um entendimento oposto do funcionalismo de que a aprendizagem é um processo adaptativo baseado na indução e dedução. Nesse, a AO sai de uma perspectiva individual para o nível coletivo. Já o paradigma crítico expõe o uso do poder e o desafio das desigualdades dentro das organizações, enfatizando a ação individual e a aprendizagem como oposto de coletivo. Nesse paradigma a aprendizagem é uma forma de adotar mudança organizacional.

É possível afirmar que cada uma das diferentes perspectivas não exclui completamente a outra, sobretudo porque muitas abordagens incorporam diferentes visões e teorias. Ainda, alguns autores, a exemplo de James March e Chris Argyris, mudaram suas perspectivas sobre aprendizagem organizacional ao longo do tempo e adicionaram novos aspectos ao fenômeno por eles estudado (PAWLOWSKY, 2001).

Logo, Popova-Nowak et al. (2015) afirmam que é possível que se apresentem pontos de conexão pela não possibilidade de estabelecer com precisão onde termina uma ênfase e começa a outra. Com isso, o olhar para o fenômeno da aprendizagem ancorado em uma única tradição pode ser substituído por uma visão holística, justificado pelo fato de que uma única forma de ver a aprendizagem possui poder de explicação limitada do fenômeno.

A interseção entre os paradigmas propostos ocorre, sobretudo, entre o funcionalista-construcionista e o construcionista-pós-moderno. Assim sendo, os estudiosos que procuram entender o fenômeno da aprendizagem organizacional têm apresentado a tendência de considerar tanto os efeitos da eficiência pregada pelo paradigma funcionalista, quanto as relações sociais que permeiam os paradigmas construcionista e pós-moderno (POPOVA-NOWAK et al., 2015). Neste sentido, acredita-se que as contribuições de autores ancorados em uma perspectiva de estudo mais funcionalista, como Cyert et al. (1963), Levitt et al. (1988), Argyris et al. $(1978,1996)$, não podem ser desconsideradas, mas podem e devem agregar outros conhecimentos como o de Brown et al. (1991); Cook et al. (1999), Elkjaer (2009), entre outros nomes, que têm uma perspectiva mais construcionista. 
Acredita-se que é possível e pertinente desenvolver estudos que se encaixam em diferentes zonas de transição dos paradigmas propostos para a AO. No entanto, não se pretende afirmar aqui que isso seja uma tarefa fácil. Mas, essa dinâmica pode proporcionar o desenvolvimento teórico do campo e, ao mesmo tempo, permitir aos estudiosos da temática adotar diferentes visões para o mesmo fenômeno, elucidando questões que não podem ser respondidas por apenas uma abordagem, perspectiva ou paradigma; como é o caso dos aspectos que estão envolvidos no processo de aprendizagem organizacional, quais sejam: pessoas, transações (relacionamentos), o contexto aonde a aprendizagem ocorre, bem como as questões relativas ao tempo e aos eventos que se desencadeiam no ambiente organizacional.

\section{Tempo e contexto em aprendizagem}

O contexto em que o aprendizado ocorre ganha importância especial ao se estudar aprendizagem organizacional, já que a aprendizagem não ocorre no vácuo, no nada, mas incorporado em uma estrutura ambiental onde as ações e os eventos se desencadeiam (ARGOTE, 2011). A ocorrência desses eventos de aprendizagem torna o tempo outra importante dimensão no processo e política de aprendizagem organizacional, uma vez que a natureza da estrutura temporal dominante impacta o progresso da aprendizagem e a ocorrência de descontinuidades (BERENDS et al., 2010).

Aproximar-se do fenômeno da aprendizagem organizacional envolve conhecer o contexto profundamente muito mais que apenas descrever os acontecimentos de uma organização. Isso implica em buscar entender a organização como um ambiente de aprendizagem, o que inclui um entendimento da relação entre indivíduos e organizações (ELKJAER, 2009) e todas as facetas que estão inseridas nesse contexto de aprendizagem, tais como poder, liderança, conflitos, entre outros aspectos.

Além disso, processos complexos como aprendizagem, inovação, mudança, entre outros processos organizacionais, requerem a necessidade de expandir a noção de tempo para melhor entender como os aspectos temporais exercem influência sobre eles (GARUD et al., 2016). Isso abrange repensar a própria percepção que se tem do tempo, incluindo a relação entre passado, presente e futuro.

No entanto, não é tarefa fácil separar totalmente tempo e contexto ao se estudar aprendizagem organizacional. Alguns estudiosos colocam o tempo como algo que faz parte do contexto, a exemplo de Salvato et al. (2011), os quais afirmam que contexto é um aspecto que engloba o ambiente, o tempo, as atividades passadas, a interação com outras pessoas e artefatos, e ainda, os estados emocional, mental e psicológico dos indivíduos. Por outro lado, alguns autores, a exemplo de Berends et al. (2010) acrescentam que a forma como o processo de aprendizagem se desdobra e como surgem as descontinuidades ao longo do tempo, pode ser influenciado pelas estruturas social e temporal. Neste caso, tempo e contexto são vistos como coisas distintas.

Sobre o contexto, Brandi et al. (2011) destacam dois entendimentos: contexto como produto histórico do qual as pessoas são parte e contexto como algo construído pela interação entre as pessoas. Já Garud et al. (2016) referem-se à complexidade relacional para se referir ao contexto onde os processos acontecem, com seu conjunto de atores sociais e elementos materiais presentes. A partir da visão da 
complexidade relacional, mudanças em uma parte de um processo impactam sobre outras partes de uma maneira interativa (GARUD et al., 2013).

Fiol et al. (1985) expõem que a cultura corporativa, a estratégia e a estrutura organizacional, além do próprio ambiente onde a organização está inserida são fatores contextuais que podem afetar a ocorrência da aprendizagem. Note-se que muitos são os fatores presentes no contexto e cada um deles pode influenciar na forma como os processos ocorrem na organização.

Strauss (1993) introduziu os conceitos de trajetória e matriz de condições (ou matriz condicional) para compreender as ações e interações que ocorrem nos mundos sociais, isto é, a vida organizacional que se desdobra entre os membros de um contexto da organização. Trajetória, para o autor, é o conceito que pode ser usado para identificar um fenômeno no tempo, de tal forma que isto pode ser entendido como um curso histórico de eventos. A descrição da trajetória se desdobra sobre certas condições que em seu turno forma a matriz condicional necessária para entender o desenvolvimento e o resultado desta trajetória. Portanto, descrever a matriz condicional significa realizar a descrição do contexto, das condições para as ações e as interações dos participantes (ELKJAER, 2009).

Assim, "as interações e os atores são afetados por diferentes combinações das condições estruturais e interacionais" (STRAUSS, 1993, 54). Conhecer toda a dinâmica da trajetória, continua o autor, pode permitir um melhor entendimento sobre o contexto em que dado fenômeno está localizado e porque as coisas acontecem de determinada forma. Trajetória está, pois, relacionada com o tempo, e a matriz condicional com o espaço onde as interações sociais se desencadeiam (ELKJAER, 2009).

No que se refere ao tempo, Reneicke et al. (2017) expõem o tempo-relógio (chronos) e o tempoprocesso (kairós) como sendo formas diferentes de ver e compreender o tempo nos estudos organizacionais. O tempo-relógio é linear, objetivo, quantificável e absoluto, o que torna o momento presente desanexado do passado e do futuro. Por outro lado, o tempo-processo é não linear, qualitativamente determinado, relativo e contextual o que possibilita a existência de múltiplos presentes, passados e futuros, ou um fluxo indivisível. Em acréscimo, Berends et al. (2010) indicam que o tempo pode ser cronológico - determinado pelo relógio e o calendário, e o tempo-evento - determinado pelo acontecimento de eventos marcantes.

A perspectiva que considera o tempo como kairós ou como um evento implica no fato de reconhecer que os processos, entre eles aprendizagem e inovação, são caracterizados por múltiplas escalas de tempo e não por uma única conceitualização linear (GARUD et al., 2016). Assim, os eventos são experimentados a partir de diferentes ritmos de tempo (GARUD et al., 2013), formando uma estrutura temporal que se estabelece e influencia o tempo dos processos organizacionais.

O tempo-evento pode ser visto também como momentos de experiências incomuns, raros ou não usuais, representando desafios únicos de aprendizagem (BECK et al., 2009; BERENDS et al., 2010). A partir deles é possível que a organização passe por um período de interpretações com vistas a reduzir a ambiguidade e a aprender como lidar com eventos similares no futuro. A aprendizagem, nesse caso, envolve o desenvolvimento de insights, conhecimento e associações entre ações do passado e a efetividade dessas ações com atos futuros (FIOL et al., 1985). Assim, embora o passado sempre pese no momento presente, ele 
permanece aberto à interpretação, sendo possível reinterpretá-lo, articulando as experiências anteriormente vividas à luz da situação corrente (HERNES et al., 2012).

Logo, eventos raros e incomuns requerem rica interpretação. A chave para aprender a partir desses eventos é vivenciá-los ricamente, buscando multiplicar vozes, perspectivas e interpretações. No entanto, muitas organizações têm dificuldade de aprender a partir de eventos raros e incomuns por causa da sua inabilidade de interpretar tais situações (BECK et al., 2009). Isso ocorre muitas vezes porque os atores que estão vivenciando uma determinada situação estão tão envolvidos que perdem a capacidade de perceber certas peculiaridades do momento vivenciado.

Ademais, é possível afirmar que aprendizagem organizacional não acontece isoladamente, mas dentro de um contexto organizacional aberto, que está em constante fluxo e busca por novos projetos, ideias, recursos e atenção (BERENDS et al., 2010). Assim, o conteúdo aprendido é específico do contexto e a aprendizagem é, ela mesma, a descoberta do que deve ser feito, quando e como fazer de acordo com as rotinas organizacionais específicas, bem como quais artefatos específicos usar, quando e como. E ainda, a inovação, como resultado do processo de aprendizagem, ocorre mais frequentemente em decorrência do lento processo envolvendo muitas inovações que evoluem e se relacionam, do que pelo surgimento pontual e revolucionário de um novo produto, serviço ou processo.

\section{CONCLUSÕES}

Ao longo deste ensaio foram levantadas algumas considerações gerais sobre o processo de aprendizagem nas organizações e da inovação como resultado desse processo, considerando como elementos relevantes para melhor compreender a temática exposta, os elementos relativos ao contexto e ao tempo.

Desde a década de 60, os estudos em Aprendizagem Organizacional (AO) apresentam um aumento crescente em termos qualitativos e quantitativos. Ao mesmo tempo passou a ser permeada por muitos conceitos e teorias, advindas da educação, da psicologia, da sociologia, da antropologia, da ciência da administração, entre outras áreas, o que a tornou uma temática interdisciplinar nos estudos organizacionais. Atualmente, a aprendizagem é reconhecida como um processo capaz de fortalecer a prática gerencial e proporcionar resultados relevantes para organização.

No que se refere ao tema inovação, esse tem sido alvo de investigação e análise como uma forma de compreender os aspectos particulares envolvidos em tal fenômeno, sendo debatido em diversos segmentos por pesquisadores que têm procurado dar suas contribuições ao longo do tempo. Todavia, é possível perceber que tais estudos acabaram resultando em muitas classificações e divisões da temática, o que torna o tema por vezes ambíguo e confuso. Desta forma, sejam nos aspectos relacionados à gestão da inovação, sejam em termos teóricos, debruçar-se sobre este fenômeno não é tarefa fácil, sendo necessário um criterioso recorte sobre qual aspecto da inovação se deseja aprofundar.

Visto que a aprendizagem ocorre situada no tempo e no espaço, torna-se relevante conhecer como se configura tal ambiente. Logo, o contexto e as interações que nele ocorrem apresentam papel importante 
no processo de aprendizagem organizacional. Especialmente porque a influência do poder interno e os fatores do contexto externo, sobretudo a pressão do ambiente (ZIETSMA et al., 2002) criam uma atmosfera de urgência em aprender e incorporar novos conhecimentos, a fim de responder às demandas desse mesmo ambiente com produtos, serviços e processos inovadores. Os pesquisadores devem, pois, atentar para captar a complexidade dessas interações, buscando guardar-se da tendência de percepção seletiva que decorrem de suas próprias suposições profissionais ou pessoais (STRAUSS, 1993).

Nesse sentido, inovação e aprendizagem, a partir de uma perspectiva de processo, podem ser abordados como uma sequência situada de atividades que se desencadeiam no tempo (LANGLEY et al., 2010). Essa visão permite um melhor entendimento do mundo, além de ser altamente relevante para as atividades gerenciais, uma vez que entender as ações que se desenvolvem sobre as interações contextuais complexas geram resultados que os modelos estáticos não são capazes de capturar e demonstrar (LANGLEY et al., 2010).

No entanto, apesar de sua pertinência para os estudos organizacionais, os estudos com perspectiva de processo que buscam elucidar questões acerca do tempo e do contexto, ainda são subvalorizados frente aos modelos conceituais baseados nos padrões tradicionais de pesquisa. Isso pode ocorrer devido à dificuldade em apreciar ou captar corretamente as questões relativas ao tempo e temporalidade, bem como de perceber todas as nuances e aspectos presentes no contexto onde ocorrem os processos.

É nessa lacuna que futuros estudos podem ser desenvolvidos, buscando produzir análises e reflexões que venham a contribuir para elucidar aspectos que ainda parecem obscuros no que se refere ao tempo e ao contexto que permeiam a aprendizagem. Tais trabalhos de pesquisa podem oferecer entendimento de como o conhecimento gerado ao longo do tempo, permeado por vários elementos contextuais, resulta em inovações que são desenvolvidas e implementadas pelas organizações. Especificamente, é possível debruçarse sobre as questões relativas ao tempo e seus eventos marcantes, ao contexto onde ocorrem os fenômenos e aos processos de aprendizagem e inovação com suas etapas e resultados. É fato que há muito a ser investigado sobre esses fenômenos. Almeja-se que pesquisas futuras expandam as bases teóricas que sustentam a temática proposta e que estes novos estudos possam permitir o avanço do campo como um todo.

\section{REFERÊNCIAS}

ANTONELLO, C. S.; GODOY, A. S.. A encruzilhada da aprendizagem organizacional: uma visão multiparadigmática. Revista de Administração

Contemporânea, Curitiba, v.14, n.2, p.310-332, 2010. DoI: http://dx.doi.org/10.1590/S1415-65552010000200008

ARAÚJO, G. D.; SILVA, A. B.; BRANDÃO, J. M. F.. O que revela a literatura internacional sobre os vínculos entre aprendizagem, competências e inovação?. Revista de Administração e Inovação, São Paulo, v.12, n.2, p.7-37, 2015. DOI: https://doi.org/10.11606/rai.v12i2.100330
ARGOTE, L.. Organizational learning research: past, present and future. Management Learning, v.42, n.4, p.439-446, 2011. DOI: http://doi.org/10.1177/1350507611408217

ARGOTE, L.; MIRON-SPEKTOR, E.. Organizational Learning: From Experience to Knowledge. Organizational Science, v.22, n.5, p.1123-1137, 2011. DOI: https://doi.org/10.1287/orsc.1100.0621

ARGYRIS, C.; SCHÖN, D. A.. Organizational Learning: A Theory of Action Perspective. Reading: Addison Wesley, 1978. 
ARGYRIS, C.; SCHÖN, D. A.. Organizational Learning II: Theory, Method and Practice. Reading: Addison Wesley, 1996.

BAILEY, J. R.; FORD, C.. Innovation and Evolution: Managing Tensions Within and Between the Domains of Theory and Practice. In: SHAVININA, L. V.. The International Handbook on Innovation. New Jersey: Elsevier Science, 2003. p.248257.

BECK, T. E.; PLOWMAN, D. A.. Experiencing Rare and Unusual Events Richly: The Role of Middle Managers in Animating and Guiding Organizational Interpretation. Organization Science, v.20, n.5, p.909-924, 2009. DOI: http://doi.org/10.1287/orsc.1090.0451

BERENDS, H.; LAMMERS, I.. Explaining discontinuity in organizational learning: a process analysis. Organization Studies, v.38, n.8, p.1045-1068, 2010. DOI: https://doi.org/10.1177/0170840610376140

BESSANT, J.. Challenges in Innovation Management. In: SHAVININA, L. V.. The International Handbook on Innovation. New Jersey: Elsevier Science, 2003.

BESSANT, J.; TIDD, J.. Inovação e empreendedorismo. Porto Alegre: Bookman, 2009.

BRANDI, U.; ELKJAER, B.. Organizational learning viewed from a social learning perspective. In: EASTERBY-SMITH, M.; LYLES, M.. Handbook of organizational learning and knowledge management. 2 ed. Chichester: John Wiley and Sons, 2011. p.23-41. DOI:

https://doi.org/10.1002/9781119207245.ch2

BROWN, J. S.; DUGUID, P.. Organizational learning and communities-of-practice: toward a unified view of working, learning, and innovation. Organization Science, v.2, n.1, p.40-57, 1991. DOI: https://doi.org/10.1287/orsc.2.1.40

BURGELMAN, R. A.; CHRISTENSEN, C. M.; WHEELWRIGHT, S. C.. Gestão estratégica da tecnologia e da inovação: conceitos e soluções. 5 ed. Porto Alegre: AMGH, 2012.

COOK, S.; BROWN, J.. Bridging epistemologies: the generative dance between organizational knowledge and organizational knowing. Organization Science, v.10, n.4, p.381-400, 1999. DOI: https://doi.org/10.1287/orsc.10.4.381

CYERT, R.; MARCH, J.. A behavioral theory of the firm. New Jersey: Prentice Hall, 1963.

DOSI, G.. Technical change and industrial transformation. New York: St. Martin's Press, 1984.

DOUGHERTY, D.. Organizando para a Inovação. In: CLEGG, S. R.; HARDY, C.; NORD, W.. Handbook de Estudos Organizacionais. São Paulo: Atlas, 2004.

EASTERBY-SMITH, M.; LYLES, M.. The evolving field of organizational learning and knowledge management. In: EASTERBY-SMITH, M.; LYLES, M.. Handbook of organizational learning and knowledge management. 2 ed. John Wiley and Sons, 2011. p.1-20. DOI: http://doi.org/10.1002/9781119207245.ch1
ELKJAER, B.. Organizational learning with a pragmatic slant. International Journal of Lifelong Education, v.22, n.5, p.481494, 2003. DOI:

https://doi.org/10.1080/0260137032000102841

ELKJAER, B.. Organizational learning: the 'third way'. Management Learning, v.35, n.4, p.419-434, 2004. DOI: https://doi.org/10.1177/1350507604048271

ELKJAER, B.. Pragmatism - A learning theory for the future. In: ILLERIS, K.. Contemporary Theories of Learning: Learning Theorists - in Their Own Words. Taylor \& Francis e-Library, 2009.

ELKJAER, B.. Pragmatismo: uma teoria da aprendizagem para o futuro. In: ILLERIS, K.. Teorias Contemporâneas da Aprendizagem. Porto Alegre: Penso, 2013. p.91-107.

FAGERBERG, J.. Innovation: A guide to the literature. In: FAGERBERG, J.; MOWERY, D. C.; NELSON, R. R.. The Oxford Handbook of Innovation. Oxford University Press, 2004. DOI:

http://doi.org/10.1093/oxfordhb/9780199286805.003.0001

FIOL, C.; LYLES, M.. Organizational learning. Academy of Management Review, v.10, n.4, p.803-813, 1985. DOI: http://doi.org/10.2307/258048

FREEMAN, C.. Innovation and long cycles of economic development. In: INTERNACIONAL SEMINAR ON INNOVATION AND DEVELOPMENT AT THE INDUSTRIAL SECTOR. Anais. Campinas: UNICAMP, 1982.

FREEMAN, C.. The Economics of Industrial Innovation. Harmondsworth: Penguin Books, 1974.

FREEMAN, C.; PEREZ, C.. Structural crisis of adjustment, business cycles and investment behaviourin. In: DOSI, G.; FREEMAN, C.; NELSON, R.; SILVERBERG, G.; SOETE, L.. Technical Change and Economic Theory. Londres: Printer, 1998. p.38-66.

FUCK, M. P.; VILHA, A. M.. Inovação Tecnológica: da definição à ação. Contemporâneos Revista de Artes e Humanidades, n.9, 2012.

GARUD, R.; DUNBAR, R. L. M.; BARTEL, C. A.. Dealing with Unusual Experiences: A Narrative Perspective on Organizational Learning. Organization Science, v.22, n.3, p.587-601, 2011a. DOI: https://doi.org/10.1287/orsc.1100.0536

GARUD, R.; DUNBAR, R. L. M.; BARTEL, C. A.; GEHMAN, J.; KUMARASWAMY, A. Complexity Arragements for Sustained Innovations: Lessons from 3M Corporation. Organization Studies, v.32, n.6, p.737-767, 2011b. DOI: https://doi.org/10.1177/0170840611410810

GARUD, R.; DUNBAR, R. L. M.; BARTEL, C. A.; TUERTSCHER, P. From the Process of Innovation to Innovation as Process. In: LANGLEY, A.; TSOUKAS, H.. The SAGE Handbook of Process Organization Studies. SAGE Publications, 2016.

GARUD, R.; DUNBAR, R. L. M.; BARTEL, C. A.; TUERTSCHER, P.; VAN DE VEN, A. H.. Perspectives on Innovation Processes. The Academy of Management Annals, v.7, n.1, p.773-817, 2013. DOI: https://doi.org/10.5465/19416520.2013.791066 
HERNES, T.; IRGENS, E.. Keeping things mindfully on track: organizational learning under continuity. Management Learning, v.44, n.3, p.253-266, 2012. DOI: https://doi.org/10.1177/1350507612445258

JIMÉNEZ-JIMÉNEZ, D.; SANZ-VALLE, R.. Innovation, organizational learning, and performance. Journal of Business Research, v.64, p.408-417, 2011. DOI: https://doi.org/10.1016/i.jbusres.2010.09.010

KNIPFER, K.; KUMP, B.; WESSEL, D.; CRESS, U.. Reflection as a catalyst for organizational learning. Studies in Continuing Education, v.35, n.1, p.30-48, 2013. DOI: http://doi.org/10.1080/0158037X.2012.683780

LANGLEY, A.; SMALLMAN, C.; TSOUKAS, H.; VAN DE VEM, A. $\mathrm{H}$.. Process studies of change in organization and management: unveiling temporality, activity, and flow. Academy of Management Journal, v.56, n.1, p.1-13, 2013. DOI: https://doi.org/10.5465/amj.2013.4001

LANGLEY, A.; SMALLMAN, C.; TSOUKAS, H.; VAN DE VEM, A. H.; TSOUKAS, H.. Introducing - Perspectives on Process Organization Studies. In: HERNES, T.; MAITLIS, S.. Process, Sensemaking, and Organizing. Oxford University Press, 2010.

LERVIK, J. E.; FAHY, K. M.; EASTERBY-SMITH, M.. Temporal dynamics of situated learning in organizations. Management Learning, v.41, n.3, p.285-301, 2010. DOI: http://doi.org/10.1177/1350507609357004

LEVITT, B.; MARCH, J.. Organizational learning. Annual Review of Sociology, v.14, p.319-340, 1988. DOI: https://doi.org/10.1146/annurev.so.14.080188.001535

NELSON, R. R.; WINTER, S. G.. Simulation of Shumpeterian competition. American Economic Review, v.67, p.271-276, 1977.

NELSON, R. R.; WINTER, S. G.. An evolutionary theory of economic change. Massachusetts: Harvard University Press, 1982.

NONAKA, I.. A dynamic theory of organizational learning creation. Organization Science, v.5, n.1, 1994. DOI: https://doi.org/10.1287/orsc.5.1.14

OECD. Organization for Economic Co-operation and Development. Manual de Oslo: Diretrizes para coleta e interpretação de dados sobre inovação. Brasília: $O E C D$, 2005.

PAWLOWSKY, P.. Management science and organizational learning. In: DIERKES, M.; BERTHOIN-ANTAL, A.; CHILD, J.; NONAKA, I.. Handbook of Organisational Learning and Knowledge. Oxford University Press, 2001.

PENG, D. X.; SCHROEDER, R. G.; SHAH, R.. Linking routines to operations capabilities: a new perspective. Journal of Operation Management, v.26, p.730-748, 2008. DOI: https://doi.org/10.1016/j.jom.2007.11.001

PEREIRA, A. J.; LOPES, H. C.. Uma perspectiva 'institucionalista evolucionária' do atraso inovativo brasileiro. In: ENCONTRO NACIONAL DE ECONOMIA POLÍTICA, 20. Anais. Foz do Iguaçu: SEP, 2015.

POPOVA-NOWAK, I.; CSEH, M.. The meaning of organizational learning: a meta-paradigm perspective. Human Resource Development Review, v.14, n.3, p.299331, 2015. DOI: http://doi.org/10.1177/1534484315596856

REINECKE, J.; ANSARI, S.. Time, Temporality, and Process Studies. In: LANGLEY, A.; TSOUKAS, H.. The SAGE Handbook of Process Organization Studies. SAGE Publications, 2017.

ROGERS, E. M.. Diffusion of innovations. 3 ed. New York: Free Press, 1983.

ROSENBERG, N.. Inside the black box: technology and economics. Cambridge: Cambridge University Press, 1982.

ROSSETTO, D. E.; BORINI, F. M.; BERNARDES, R. C.. Estrutura e evolução da Inovação através da análise da literatura dos últimos 60 anos. In: SIMPÓSIO DE ADMINISTRAÇÃO DA PRODUÇÃO, LOGÍSTICA E OPERAÇÕES INTERNACIONAIS, 19. Anais. São Paulo: FGV, 2016.

SALVATO, C.; RERUP, C.. Beyond collective entities: Multilevel research on organizational routines and capabilities. Journal of Management, v.37, n.2, p.468-490, 2011. DOI: https://doi.org/10.1177/0149206310371691

SOUZA, N. J.. Desenvolvimento Econômico. São Paulo: Atlas, 2005.

STRAUSS, A.. Continual permutations of action. New Brunswick: Transaction Publishers, 1993.

SWAN, J.; SCARBOROUGH, H.; ROBERTSON, M.. Linking knowledge, Networking and Innovation Processes: A Conceptual Model. In: SHAVININA, L. V. The International Handbook on Innovation. New Jersey: Elsevier, Science, 2003. DOI: http://doi.org/10.1016/B978-0080441986/50046-2

SZMRECSÁNYI, T.. Ideias Fundadoras: Apresentação. Revista Brasileira de Inovação, v.1, n.2, 2002. DOI: https://doi.org/10.20396/rbi.v1i2.8648859

TEECE, D. J.. Explicating dynamic capabilities: The nature and microfoundations of (sustainable) enterprise performance. Strategic Management Journal, v.28, p.1319-1350, 2007. DOI: https://doi.org/10.1002/smj.640

TIDD, J.; BESSANT, J.; PAVITT, K.. Gestão para inovação. 3 ed. Porto Alegre: Bookman, 2008.

TIGRE, P.. Gestão da inovação: a economia da tecnologia no Brasil. Rio de Janeiro: Elsevier, 2006.

TORNATZKY, L. G.; FLEISCHER, M.. The processes of Technological Innovation. Toronto: Lexington Books, 1990.

TSOUKAS, H.. A Dialogical Approach to the Creation of New Knowledge in Organizations. Organization Science, v.20, n.6, p.941-957, 2009. DOI:

https://doi.org/10.1287/orsc.1090.0435 
VAN DE VEN, A. H.. Central problem in the Management of Innovation. Management Science, v.32, n.5, p.590-607, 1986. DOI: https://doi.org/10.1287/mnsc.32.5.590

VAN DE VEN, A. H.. Suggestions for studying strategy process: A research note. Strategic Management Journal, v.13, p.169-188, 1992. DOI: https://doi.org/10.1002/smj.4250131013

VAN DE VEN, A. H.; POOLE, M. S.. Methods for Studying Innovation Development in the Minnesota innovation
Research Program. In: HUBER, G. P.; VAN DE VEN, A. H.. Longitudinal Field Research Methods: Studying processes of organizational change. Sage Publications, 1995. DOI: https://doi.org/10.1287/orsc.1.3.313

ZIETSMA, C.; WINN, M.; BRANZEI, O.; VERTINSKY, I.. The War of the Woods: Facilitators and Impediments of Organizational Learning Processes. British Journal of Management, v.13, p.S61-S74, 2002. DOI: https://doi.org/10.1111/1467-8551.13.s2.6

A CBPC - Companhia Brasileira de Produção Científica (CNPJ: 11.221.422/0001-03) detém os direitos materiais desta publicação. Os direitos referem-se à publicação do trabalho em qualquer parte do mundo, incluindo os direitos às renovações, expansões e disseminações da contribuição, bem como outros direitos subsidiários. Todos os trabalhos publicados eletronicamente poderão posteriormente ser publicados em coletâneas impressas sob coordenação da Sustenere Publishing, da Companhia Brasileira de Produção Científica e seus parceiros autorizados. Os (as) autores (as) preservam os direitos autorais, mas não têm permissão para a publicação da contribuição em outro meio, impresso ou digital, em português ou em tradução. 